\title{
Multi-Objective Optimization Study for the Turnout Sub-Rail Parameters Based on Orthogonal Experiment
}

\author{
Lu Yongjie ${ }^{*}, 1,2$, Zhu Bowen ${ }^{3}$, Wang Jianxi ${ }^{4}$

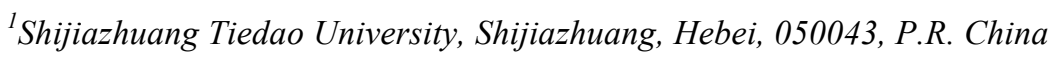 \\ ${ }^{2}$ Key Laboratory of Traffic Safety and Control in Hebei, Shijiazhuang, Hebei, 050043, P.R. China \\ ${ }^{3}$ Taiyuan Railway Transportation Equipment CO., LTD, Taiyuan, Shanxi, 030009, P.R. China \\ ${ }^{4}$ Key Laboratory of Road and Railway Engineering Safety Control of Ministry of Education (Shijiazhuang Tiedao \\ University), Shijiazhuang, Hebei, 050043, P.R. China
}

\begin{abstract}
The stiffness and damping characteristics under the turnout rail are important factors affecting vehicle safety and stable running. According to the variable cross-section structure of a single $60 \mathrm{~kg} / \mathrm{m}$ turnout No.18, a multi-body dynamic model of the vehicle-turnout coupled system is established. Based on the orthogonal test method, 25 group working conditions have been designed with the combination of the sub-rail stiffness and damping parameters. It has been found that the stiffness parameters under the rail have a greater impact on the vehicle's performance through the range analysis of the simulation results. The vertical sub-rail stiffness mainly influences the vehicle's vertical response and Sperling indicator. The lateral stiffness affects the derailment coefficient and the riding comfort. The matching scheme of the sub-rail stiffness and damping parameters is deduced by aiming towards the optimal vehicle security, stability and comfort, which provides a reference for the sub-rail structure design.
\end{abstract}

Keywords: Multi-body dynamic, Orthogonal test, Sub-rail, Turnout, Vehicle, Orthogonal scheme, Optimization study.

\section{INTRODUCTION}

Turnout has a complicated structure, which is the key component of the railway track. Vibrations and shocks exist between the wheel and the turnout due to the complicated and mutable wheel-rail contact geometry when a vehicle is passing through the turnout. That is why the turnout, the curve and the joint are called the three weakest links of railway track [1]. Because of the particular structure of turnout, the relationship between the track rigidity and the damping is complex. The track's mechanical parameters are important influencing factors on the service life of the turnout. However, the turnout life is also affected by its maintenance work and its dynamic behavior when a vehicle is passing through the turnout. Therefore, the reasonable value of track rigidity and damping has a great significance towards the dynamics performance of wheel-turnout. However, more and more attention has been paid to the optimization analysis of the track vertical stiffness by scholars at home and abroad. A finite element calculation model of the track has been built according to the $60 \mathrm{~kg} / \mathrm{m}$ No.12 speed-raising single-pointed turnout by Tan Xiaochun [2]. The distribution regularity of integral rigidity of turnouts has been obtained and the homogenization measure has been put forward to further optimize the track rigidity for train/frog system. To identify the variation rule for track rigidity of a turnout lying on a ballast less track, Chen

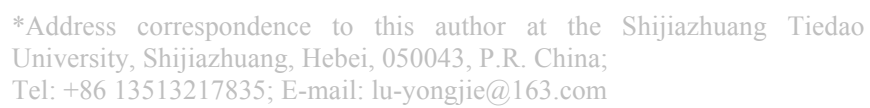
University, Shijiazhuang, Hebei, 050043, P.R. China;
Tel: +86 13513217835; E-mail: lu-yongjie@163.com
Xiaoping established a calculation model for Chinese No.12 speeding-up turnout for track rigidity based on FEM [3]. The objective was to study the homogenization of track rigidity. With the theoretical foundation of the turnout rigidity optimized, a dynamics simulation model of vehicle-rail relationship was established to investigate the effect between the dynamics characteristics of wheel-rail contact model and track vertical rigidity based on rigid-flexible coupling system by $\mathrm{Fu}$ Yifan [4]. Klaus Knothe and Karl Popp in a study pointed out the influence of track rigidity based on the system dynamics and long-term behavior of vehicle, track and sub grade [5].

In this paper, a trailer model of $\mathrm{CRH} 2 \mathrm{C}$ Electric Multiple Unit and the $60 \mathrm{~kg} / \mathrm{m}$ Rail Chinese NO.18 turnout with a movable-nose model have been established. By using the orthogonal method, four factors are discussed, such as track vertical and lateral rigidity and damping, which affects the dynamics performance of the turnout when a vehicle passes through the turnout using the simulation analysis. The optimal matching parameters of the rigidity and damping are shown by using the range analysis.

\section{DYNAMICS MODEL OF VEHICLE-TURNOUT}

\subsection{Turnout Model}

According to the variable cross-section characteristics, the $60 \mathrm{~kg} / \mathrm{m}$ Rail Chinese NO.18 turnout with a movablenose model is established using the multi-body dynamics analysis software. According to reference [6], the turnout is designed with a total length of $69 \mathrm{~m}$. The curve radius is $1100 \mathrm{~m}$, the crossing angle is $3^{\circ} 10^{\prime} 47.39^{\prime \prime}$, the track gauge is 
$1435 \mathrm{~mm}$, and the measurement point of track gauge is $0.016 \mathrm{~m}$, the canter distance of the tracks is $1508 \mathrm{~mm}$. The length of the tongue rail is $20.97 \mathrm{~m}$ and the length of the frog is $10.2 \mathrm{~m}$.

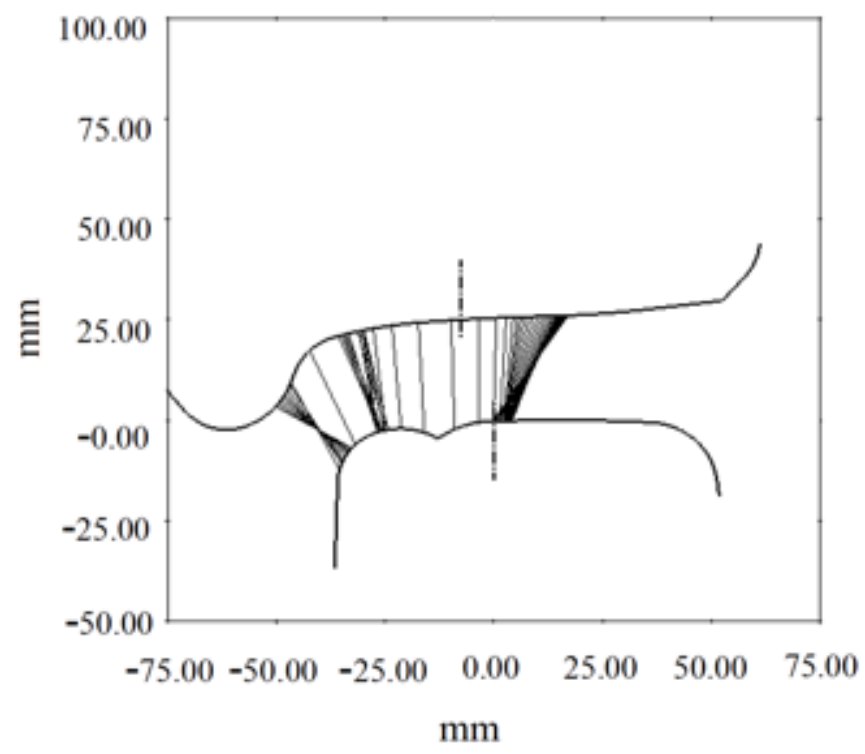

Fig. (1). Geometric contact relationship between LM treads and switch rail.

Several main characteristics of the cross-sections of the turnout are stock rail, tongue rail and stock rail with close, long and short nose rails. The silhouettes of characteristic cross-sections were rendered in 2D graphic software. Then data files were constructed by discrete points of the different profile cross-sections. The strategy was to cover the whole track surface in order to interpolate the rail profile between the two measured cross-sections by connecting corresponding discrete points on each cross-section. The structures of turnout can be listed as follows: stock rails, guard rail, switch proper and frog. 15 profile cross-sections were constructed to describe the structures. When the vehicle is passing through the turnout on the divergent route, 4 rail assembly files have to be generated - 2 for the contact between the tread/flange of a wheel and the appropriate rails and 2 for the contact between the back of a wheel and the rail. The geometric contact relationship between tongue rail with top width of $30 \mathrm{~mm}$ and wheel track with LM type treads is presented in Fig. (1).

\subsection{Vehicle Model}

On the basis of reference literature [7], a trailer model of CRH2C Electric Multiple Unit was established with the LM type treads and wheel diameter $0.86 \mathrm{~m}$. The secondary suspension model was maintained by an air spring, a lateral damper and a draw bar. The composition of the primary suspension was a primary spring and vertical damper. The dynamic simulation looked like a car body, and frame and wheel set were designed as a rigid body. Meanwhile, lateral stop and anti-simulation damper were considered as the nonlinear force element. The diagram (Fig. 2) shows a dynamics vehicle model.

\section{ORTHOGONAL DESIGN}

The Orthogonal design method has been extensively used in manufacturing and other fields. It is used to assess relatively best experimental conditions and production processes with reference to the orthogonal designs table and representative factors to investigate the optimum results $[8$, 9]. This article gives the combination of track vertical and lateral rigidity, damping through the orthogonal experiment analysis with four factors in five levels according to the references [10], as shown in Table 1.

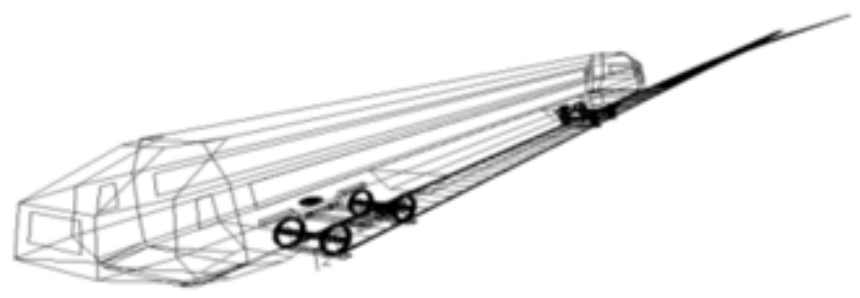

Fig. (2). Multi-body dynamics model of vehicles-turnout.

Table 1. Orthogonal scheme when vehicle passes through turnout.

\begin{tabular}{|c|c|c|c|c|}
\hline $\begin{array}{c}\text { Experimental } \\
\text { Condition }\end{array}$ & $\begin{array}{c}\boldsymbol{K}_{\mathbf{V}} \\
(\mathbf{N} / \mathbf{m})\end{array}$ & $\begin{array}{c}C_{\mathrm{V}} \\
(\mathrm{Ns} / \mathrm{m})\end{array}$ & $\begin{array}{c}\boldsymbol{K}_{\mathrm{H}} \\
(\mathbf{N} / \mathbf{m})\end{array}$ & $\begin{array}{c}C_{\mathrm{H}} \\
(\mathrm{Ns} / \mathrm{m})\end{array}$ \\
\hline 1 & $\mathrm{~L} 1=2 \times 10^{7}$ & $\mathrm{~L} 1=1 \times 10^{4}$ & $\mathrm{~L} 1=1 \times 10^{7}$ & $\mathrm{~L} 1=1 \times 10^{4}$ \\
\hline 2 & $\mathrm{~L} 1=2 \times 10^{7}$ & $\mathrm{~L} 2=3.25 \times 10^{4}$ & $\mathrm{~L} 2=1.5 \times 10^{7}$ & $\mathrm{~L} 2=2 \times 10^{4}$ \\
\hline 3 & $\mathrm{~L} 1=2 \times 10^{7}$ & $\mathrm{~L} 3=5.5 \times 10^{4}$ & L3 $=2 \times 10^{7}$ & L3 $=3 \times 10^{4}$ \\
\hline 4 & $\mathrm{~L} 1=2 \times 10^{7}$ & L4 $=7.75 \times 10^{4}$ & $\mathrm{~L} 4=2.5 \times 10^{7}$ & $\mathrm{~L} 4=4 \times 10^{4}$ \\
\hline 5 & $\mathrm{~L} 1=2 \times 10^{7}$ & $\mathrm{~L} 5=10 \times 10^{4}$ & $\mathrm{~L} 5=3 \times 10^{7}$ & $\mathrm{~L} 5=5 \times 10^{4}$ \\
\hline 6 & $\mathrm{~L} 2=3.5 \times 10^{7}$ & $\mathrm{~L} 1=1 \times 10^{4}$ & $\mathrm{~L} 2=1.5 \times 10^{7}$ & L3 $3=3 \times 10^{4}$ \\
\hline 7 & $\mathrm{~L} 2=3.5 \times 10^{7}$ & $\mathrm{~L} 2=3.25 \times 10^{4}$ & $\mathrm{~L} 3=2 \times 10^{7}$ & $\mathrm{~L} 4=4 \times 10^{4}$ \\
\hline 8 & $\mathrm{~L} 2=3.5 \times 10^{7}$ & $\mathrm{~L} 3=5.5 \times 10^{4}$ & $\mathrm{~L} 4=2.5 \times 10^{7}$ & L $5=5 \times 10^{4}$ \\
\hline 9 & $\mathrm{~L} 2=3.5 \times 10^{7}$ & L4 $=7.75 \times 10^{4}$ & $\mathrm{~L} 5=3 \times 10^{7}$ & $\mathrm{~L} 1=1 \times 10^{4}$ \\
\hline 10 & $\mathrm{~L} 2=3.5 \times 10^{7}$ & $\mathrm{~L} 5=10 \times 10^{4}$ & $\mathrm{~L} 1=1 \times 10^{7}$ & $\mathrm{~L} 2=2 \times 10^{4}$ \\
\hline 11 & $\mathrm{~L} 3=5 \times 10^{7}$ & $\mathrm{~L} 1=1 \times 10^{4}$ & $\mathrm{~L} 3=2 \times 10^{7}$ & $\mathrm{~L} 5=5 \times 10^{4}$ \\
\hline 12 & $\mathrm{~L} 3=5 \times 10^{7}$ & $\mathrm{~L} 2=3.25 \times 10^{4}$ & $\mathrm{~L} 4=2.5 \times 10^{7}$ & $\mathrm{~L} 1=1 \times 10^{4}$ \\
\hline 13 & $\mathrm{~L} 3=5 \times 10^{7}$ & $\mathrm{~L} 3=5.5 \times 10^{4}$ & $\mathrm{~L} 5=3 \times 10^{7}$ & $\mathrm{~L} 2=2 \times 10^{4}$ \\
\hline 14 & $\mathrm{~L} 3=5 \times 10^{7}$ & L4 $=7.75 \times 10^{4}$ & $\mathrm{~L} 1=1 \times 10^{7}$ & L3 $3=3 \times 10^{4}$ \\
\hline 15 & $\mathrm{~L} 3=5 \times 10^{7}$ & $\mathrm{~L} 5=10 \times 10^{4}$ & $\mathrm{~L} 2=1.5 \times 10^{7}$ & $\mathrm{~L} 4=4 \times 10^{4}$ \\
\hline 16 & $\mathrm{~L} 4=6.5 \times 10^{7}$ & $\mathrm{~L} 1=1 \times 10^{4}$ & $\mathrm{~L} 4=2.5 \times 10^{7}$ & $\mathrm{~L} 2=2 \times 10^{4}$ \\
\hline 17 & $\mathrm{~L} 4=6.5 \times 10^{7}$ & $\mathrm{~L} 2=3.25 \times 10^{4}$ & $\mathrm{~L} 5=3 \times 10^{7}$ & $\mathrm{~L} 3=3 \times 10^{4}$ \\
\hline 18 & $\mathrm{~L} 4=6.5 \times 10^{7}$ & $\mathrm{~L} 3=5.5 \times 10^{4}$ & $\mathrm{~L} 1=1 \times 10^{7}$ & $\mathrm{~L} 4=4 \times 10^{4}$ \\
\hline 19 & $\mathrm{~L} 4=6.5 \times 10^{7}$ & L4 $=7.75 \times 10^{4}$ & $\mathrm{~L} 2=1.5 \times 10^{7}$ & $\mathrm{~L} 5=5 \times 10^{4}$ \\
\hline 20 & $\mathrm{~L} 4=6.5 \times 10^{7}$ & $\mathrm{~L} 5=10 \times 10^{4}$ & $\mathrm{~L} 3=2 \times 10^{7}$ & $\mathrm{~L} 1=1 \times 10^{4}$ \\
\hline 21 & $\mathrm{~L} 5=8 \times 10^{7}$ & $\mathrm{~L} 1=1 \times 10^{4}$ & $\mathrm{~L} 5=3 \times 10^{7}$ & $\mathrm{~L} 4=4 \times 10^{4}$ \\
\hline 22 & $\mathrm{~L} 5=8 \times 10^{7}$ & $\mathrm{~L} 2=3.25 \times 10^{4}$ & $\mathrm{~L} 1=1 \times 10^{7}$ & $\mathrm{~L} 5=5 \times 10^{4}$ \\
\hline 23 & $\mathrm{~L} 5=8 \times 10^{7}$ & $\mathrm{~L} 3=5.5 \times 10^{4}$ & $\mathrm{~L} 2=1.5 \times 10^{7}$ & $\mathrm{~L} 1=1 \times 10^{4}$ \\
\hline 24 & $\mathrm{~L} 5=8 \times 10^{7}$ & $\mathrm{~L} 4=7.75 \times 10^{4}$ & $\mathrm{~L} 3=2 \times 10^{7}$ & $\mathrm{~L} 2=2 \times 10^{4}$ \\
\hline 25 & $\mathrm{~L} 5=8 \times 10^{7}$ & $\mathrm{~L} 5=10 \times 10^{4}$ & $\mathrm{~L} 4=2.5 \times 10^{7}$ & $\mathrm{~L} 3=3 \times 10^{4}$ \\
\hline
\end{tabular}

There are five levels in each factor, track vertical rigidity $2 \sim 8 \times 10^{7} \mathrm{~N} / \mathrm{m}$, track vertical damping $1 \sim 10 \times 10^{4} \mathrm{Ns} / \mathrm{m}$, track lateral rigidity $1 \sim 3 \times 10^{7} \mathrm{~N} / \mathrm{m}$, track lateral damping $1 \sim 5 \times 10^{4} \mathrm{Ns} / \mathrm{m}$. 
4. SIMULATION OF THE VEHICLE DYNAMICS 4.1. PERFORMANCES

It was calculated that the axle load was 10.2495 t. The dynamics performances were simulated. The vehicle passed through the $60 \mathrm{~kg} / \mathrm{m}$ Rail Chinese NO.18 turnout at the speed of $80 \mathrm{~km} / \mathrm{h}$ on the divergent route without track spectrum. Through 25 orthogonal experiments, multi-body dynamics analysis software was applied to simulate the train running safety, operational stability and riding quality.

The derailment coefficient and wheel unloading rate are two quantitative standards for evaluating running safety of the train, which is a criterion of China railway department. The assessment criteria for the train's operational stability are made up of Sperling value and car body vibration accelerations in China. In the study, the main technical parameters were detailed as follows: Y_ws for lateral wheel set force, $Q_{-w}$ for vertical wheel-rail force, $Y / Q$ for derailment coefficient, $\Delta p / p$ for wheel unloading rate, Yacc for lateral car body vibration accelerations, $Z_{\text {acc }}$ for vertical car body vibration accelerations, Ys for lateral Sperling value, and $Z_{\mathrm{s}}$ for vertical Sperling value. The simulation time was $25 \mathrm{~s}$, and the sampling frequency was $256 \mathrm{~Hz}$. The maximum value of vehicle dynamics performances with four factors in five levels is shown in Table 2.

Table 2. Maximum value of vehicle dynamics performances with four factors of five levels.

\begin{tabular}{|c|c|c|c|c|}
\hline Intervention & \multicolumn{4}{|c|}{ Running Safety } \\
\hline Experimental Condition & $Y_{-} w s(\mathrm{kN})$ & $Q w(\mathrm{kN})$ & $Y / Q$ & $\frac{\Delta p}{\bar{p}}$ \\
\hline 1 & 17.021 & 64.059 & 0.176 & 0.244 \\
\hline 2 & 17.428 & 63.359 & 0.187 & 0.230 \\
\hline 3 & 18.064 & 62.913 & 0.194 & 0.222 \\
\hline 4 & 18.334 & 62.375 & 0.198 & 0.211 \\
\hline 5 & 18.392 & 61.862 & 0.201 & 0.201 \\
\hline 6 & 17.879 & 73.753 & 0.189 & 0.432 \\
\hline 7 & 18.387 & 72.179 & 0.194 & 0.401 \\
\hline 8 & 18.615 & 72.627 & 0.200 & 0.410 \\
\hline 9 & 18.743 & 72.365 & 0.202 & 0.405 \\
\hline 10 & 17.016 & 70.930 & 0.174 & 0.377 \\
\hline 11 & 18.592 & 86.615 & 0.199 & 0.682 \\
\hline 12 & 18.954 & 83.745 & 0.201 & 0.626 \\
\hline 13 & 18.881 & 83.890 & 0.204 & 0.629 \\
\hline 14 & 17.177 & 82.419 & 0.178 & 0.600 \\
\hline 15 & 18.021 & 81.646 & 0.188 & 0.585 \\
\hline 16 & 19.061 & 95.596 & 0.200 & 0.856 \\
\hline 17 & 18.889 & 95.489 & 0.203 & 0.854 \\
\hline 18 & 17.365 & 93.368 & 0.180 & 0.813 \\
\hline
\end{tabular}

(Table 2) contd.....

\begin{tabular}{|c|c|c|c|c|}
\hline Intervention & \multicolumn{4}{|c|}{ Running Safety } \\
\hline Experimental Condition & $\boldsymbol{Y} \_\boldsymbol{w} \mathbf{( k N )}$ & $\boldsymbol{Q} \_\boldsymbol{w}(\mathbf{k N )}$ & $\boldsymbol{V} \boldsymbol{Q}$ & $\frac{\Delta p}{\bar{p}}$ \\
\hline \hline 19 & 18.165 & 90.366 & 0.190 & 0.755 \\
\hline 20 & 18.690 & 87.859 & 0.195 & 0.706 \\
\hline 21 & 19.019 & 103.334 & 0.208 & 1.006 \\
\hline 22 & 17.495 & 102.061 & 0.180 & 0.982 \\
\hline 23 & 18.499 & 99.490 & 0.192 & 0.932 \\
\hline 24 & 18.789 & 95.418 & 0.196 & 0.853 \\
\hline 25 & 18.951 & 95.188 & 0.200 & 0.848 \\
\hline
\end{tabular}

(2-2)

\begin{tabular}{|c|c|c|c|c|c|}
\hline Intervention & \multicolumn{4}{|c|}{ Operational Stability } & \multirow{2}{*}{$\begin{array}{c}\begin{array}{r}\text { Riding } \\
\text { Quality }\end{array} \\
N_{M V}\end{array}$} \\
\hline $\begin{array}{c}\text { Experimental } \\
\text { Condition }\end{array}$ & $\operatorname{Yacc}\left(\mathbf{m} / \mathbf{s}^{2}\right)$ & $\operatorname{Zacc}\left(\mathrm{m} / \mathrm{s}^{2}\right)$ & YS & ZS & \\
\hline 1 & 0.640 & 0.125 & 0.956 & 0.323 & 1.605 \\
\hline 2 & 0.654 & 0.123 & 0.950 & 0.322 & 1.595 \\
\hline 3 & 0.658 & 0.118 & 0.945 & 0.319 & 1.588 \\
\hline 4 & 0.657 & 0.115 & 0.938 & 0.314 & 1.589 \\
\hline 5 & 0.652 & 0.112 & 0.946 & 0.310 & 1.588 \\
\hline 6 & 0.667 & 0.180 & 0.940 & 0.343 & 1.597 \\
\hline 7 & 0.664 & 0.170 & 0.937 & 0.333 & 1.592 \\
\hline 8 & 0.661 & 0.161 & 0.934 & 0.327 & 1.586 \\
\hline 9 & 0.663 & 0.155 & 0.933 & 0.321 & 1.585 \\
\hline 10 & 0.643 & 0.149 & 0.941 & 0.319 & 1.602 \\
\hline 11 & 0.669 & 0.215 & 0.940 & 0.320 & 1.592 \\
\hline 12 & 0.674 & 0.196 & 0.935 & 0.321 & 1.591 \\
\hline 13 & 0.664 & 0.176 & 0.928 & 0.321 & 1.583 \\
\hline 14 & 0.647 & 0.163 & 0.943 & 0.320 & 1.605 \\
\hline 15 & 0.667 & 0.157 & 0.942 & 0.319 & 1.598 \\
\hline 16 & 0.683 & 0.243 & 0.938 & 0.316 & 1.594 \\
\hline 17 & 0.670 & 0.216 & 0.939 & 0.314 & 1.587 \\
\hline 18 & 0.651 & 0.197 & 0.944 & 0.312 & 1.601 \\
\hline 19 & 0.670 & 0.182 & 0.938 & 0.313 & 1.597 \\
\hline 20 & 0.680 & 0.176 & 0.930 & 0.312 & 1.588 \\
\hline 21 & 0.676 & 0.219 & 0.931 & 0.316 & 1.589 \\
\hline 22 & 0.653 & 0.262 & 0.944 & 0.312 & 1.603 \\
\hline 23 & 0.696 & 0.239 & 0.939 & 0.311 & 1.599 \\
\hline 24 & 0.680 & 0.222 & 0.934 & 0.311 & 1.590 \\
\hline 25 & 0.674 & 0.206 & 0.930 & 0.310 & 1.586 \\
\hline
\end{tabular}


The sum and average of dynamics performances with each factor in different levels are shown in (Table 3).

Table 3. The average of dynamics performances with each factor.

\begin{tabular}{|c|c|c|c|c|c|}
\hline Evaluation Index & & A & B & C & D \\
\hline \multirow{5}{*}{$Y \_w s$} & $\overline{K 1}$ & 17.848 & 18.314 & 17.215 & 18.381 \\
\hline & $\overline{K 2}$ & 18.128 & 18.231 & 17.998 & 18.235 \\
\hline & $\overline{K 3}$ & 18.325 & 18.285 & 18.504 & 18.192 \\
\hline & $\overline{K 4}$ & 18.434 & 18.242 & 18.783 & 18.225 \\
\hline & $\overline{K 5}$ & 18.551 & 18.214 & 18.785 & 18.252 \\
\hline \multirow{5}{*}{$Q_{-} w$} & $\overline{K 1}$ & 62.914 & 84.671 & 82.567 & 81.504 \\
\hline & $\overline{K 2}$ & 72.371 & 83.367 & 81.723 & 81.838 \\
\hline & $\overline{K 3}$ & 83.663 & 82.458 & 80.997 & 81.952 \\
\hline & $\overline{K 4}$ & 92.536 & 80.589 & 81.906 & 82.580 \\
\hline & $\overline{K 5}$ & 99.098 & 79.497 & 83.388 & 82.706 \\
\hline \multirow{5}{*}{$Y / Q$} & $\overline{K 1}$ & 0.191 & 0.194 & 0.178 & 0.193 \\
\hline & $\overline{K 2}$ & 0.192 & 0.193 & 0.189 & 0.192 \\
\hline & $\overline{K 3}$ & 0.194 & 0.194 & 0.196 & 0.193 \\
\hline & $\overline{K 4}$ & 0.194 & 0.193 & 0.200 & 0.194 \\
\hline & $\overline{K 5}$ & 0.195 & 0.192 & 0.204 & 0.194 \\
\hline \multirow{5}{*}{$\Delta p / \bar{p}$} & $\overline{K 1}$ & 0.222 & 0.644 & 0.603 & 0.583 \\
\hline & $\overline{K 2}$ & 0.405 & 0.619 & 0.587 & 0.589 \\
\hline & $\overline{K 3}$ & 0.624 & 0.60192 & 0.573 & 0.591 \\
\hline & $\overline{K 4}$ & 0.797 & 0.565 & 0.590 & 0.603 \\
\hline & $\overline{K 5}$ & 0.924 & 0.543 & 0.619 & 0.606 \\
\hline \multirow{5}{*}{ yacc } & $\overline{K 1}$ & $\begin{array}{c}0.6527 \\
2\end{array}$ & 0.667 & 0.647 & 0.671 \\
\hline & $\overline{K 2}$ & 0.660 & 0.663 & 0.671 & 0.665 \\
\hline & $\overline{K 3}$ & 0.664 & 0.666 & 0.670 & 0.663 \\
\hline & $\overline{K 4}$ & 0.671 & 0.663 & 0.670 & 0.663 \\
\hline & $\overline{K 5}$ & 0.676 & 0.663 & 0.665 & 0.661 \\
\hline \multirow{5}{*}{ zacc } & $\overline{K 1}$ & 0.119 & 0.196 & 0.179 & 0.178 \\
\hline & $\overline{K 2}$ & 0.163 & 0.193 & 0.176 & 0.183 \\
\hline & $\overline{K 3}$ & 0.181 & 0.178 & 0.180 & 0.177 \\
\hline & $\overline{K 4}$ & 0.203 & 0.167 & 0.184 & 0.172 \\
\hline & $\overline{K 5}$ & 0.230 & 0.160 & 0.176 & 0.186 \\
\hline
\end{tabular}

(Table 3) contd......

\begin{tabular}{|c|c|c|c|c|c|}
\hline Evaluation Index & & $\mathbf{A}$ & B & C & D \\
\hline \multirow{5}{*}{$y \mathrm{~S}$} & $\overline{K 1}$ & 0.947 & 0.941 & 0.946 & 0.939 \\
\hline & $\overline{K 2}$ & 0.937 & 0.941 & 0.942 & 0.938 \\
\hline & $\overline{K 3}$ & 0.938 & 0.938 & 0.937 & 0.939 \\
\hline & $\overline{K 4}$ & 0.938 & 0.937 & 0.935 & 0.938 \\
\hline & $\overline{K 5}$ & 0.936 & 0.938 & 0.935 & 0.940 \\
\hline \multirow{5}{*}{$z \mathrm{~S}$} & $\overline{K 1}$ & 0.318 & 0.324 & 0.317 & 0.318 \\
\hline & $\overline{K 2}$ & 0.329 & 0.320 & 0.322 & 0.318 \\
\hline & $\overline{K 3}$ & 0.320 & 0.318 & 0.319 & 0.321 \\
\hline & $\overline{K 4}$ & 0.313 & 0.316 & 0.318 & 0.319 \\
\hline & $\overline{K 5}$ & 0.312 & 0.314 & 0.316 & 0.316 \\
\hline \multirow{5}{*}{$N_{M V}$} & $\overline{K 1}$ & 1.593 & 1.595 & 1.603 & 1.594 \\
\hline & $\overline{K 2}$ & 1.592 & 1.594 & 1.597 & 1.593 \\
\hline & $\overline{K 3}$ & 1.594 & 1.591 & 1.590 & 1.593 \\
\hline & $\overline{K 4}$ & 1.593 & 1.593 & 1.589 & 1.594 \\
\hline & $\overline{K 5}$ & 1.593 & 1.592 & 1.586 & 1.593 \\
\hline
\end{tabular}

The average value of dynamics performances with each factor is investigated by range analysis in Fig. (3).

By the range analysis, the relative optimum track mechanical parameters of the four factors and their parametric sensitivity have been determined in Table 4.

The simulation results above revealed that it is advantageous to select A1B5 in track vertical mechanical parameters and $\mathrm{C} 1$ or $\mathrm{C} 5$ in Track lateral rigidity. Besides the D4, other parameters of track lateral damping were available. Track rigidity has more significant influence on vehicle dynamic performances than track lateral damping. Track vertical rigidity has an effect on vertical wheel-rail force, wheel unloading rate, vertical car body vibration acceleration and lateral and vertical Sperling value. Track lateral rigidity exerts influence on lateral wheel set force, derailment coefficient, lateral car body vibration accelerations and ride comfort index.

\section{OPTIMIZATION COMPARISON}

Dynamics performances of the vehicles on the condition that the $60 \mathrm{~kg} / \mathrm{m}$ Rail Chinese NO.18 turnout with a movable-nose on the divergent route at the speed of 10$80 \mathrm{~km} / \mathrm{h}$ with the average and optimum of track mechanical parameters (Table 5) are obtained by simulation calculation and compared in Fig. (4).

A comparison between the average and optimum of track mechanical parameters simulation model shows that much improvement is obtained from the optimized design. Vehicles can drive through the turnout with best vertical wheel-rail force, wheel unloading rate and vertical car body vibration accelerations on the condition of the optimized design. And lateral car body 
vibration accelerations and ride comfort index are basically the same.

(a) Sensitivity analysis of sub-rail parameter on axle lateral force

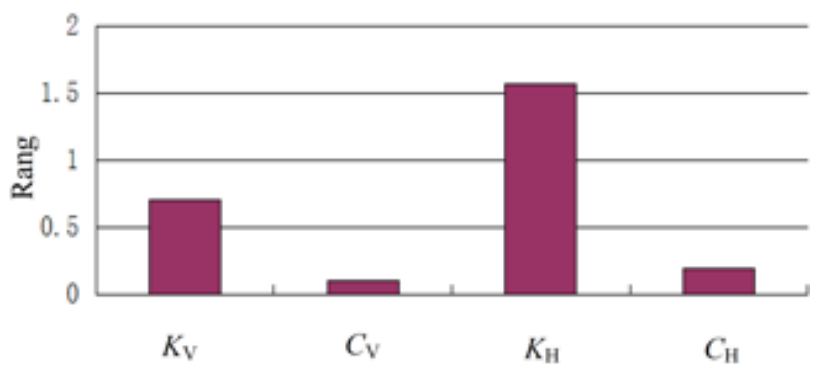

(b) Sensitivity analysis of sub-rail parameter on vertical wheel-rail force

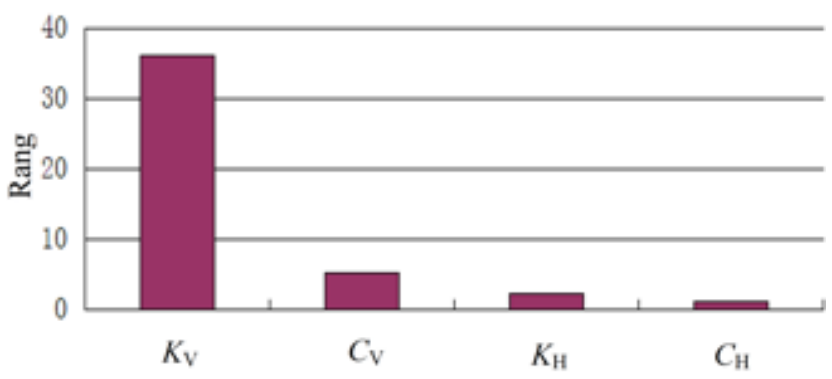

(c) Sensitivity analysis of sub-rail parameter on derailment coefficient

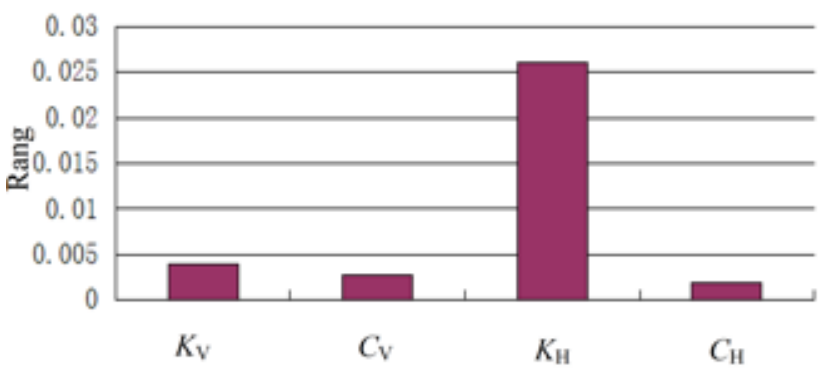

(d) Sensitivity analysis of sub-rail parameter on wheel unloading rate

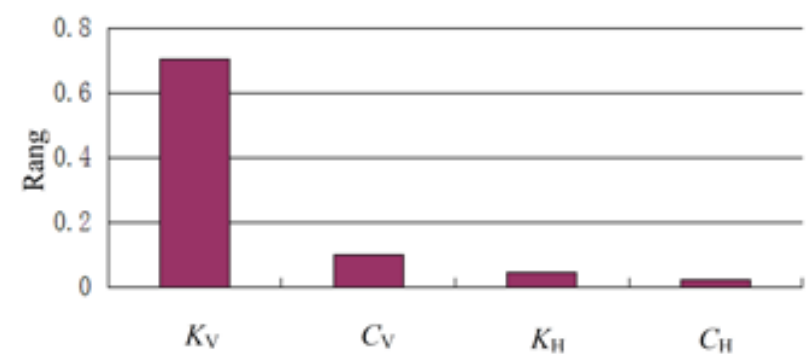

(e) Sensitivity analysis of sub-rail parameter on vehicle lateral acceleration

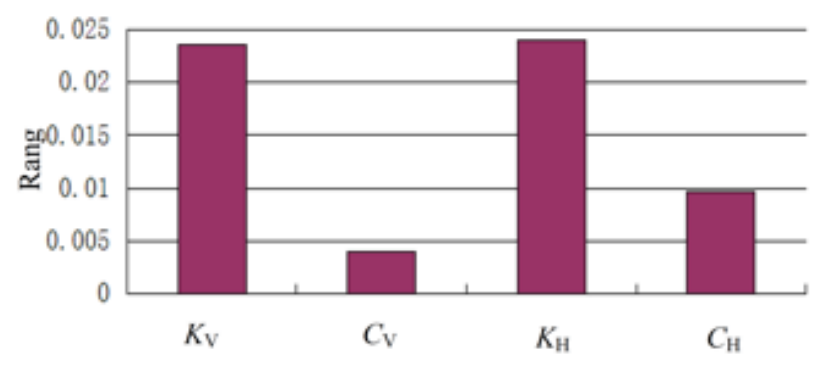

(Fig. 3) contd..... (f) Sensitivity analysis of sub-rail parameter on vehicle vertical acceleration

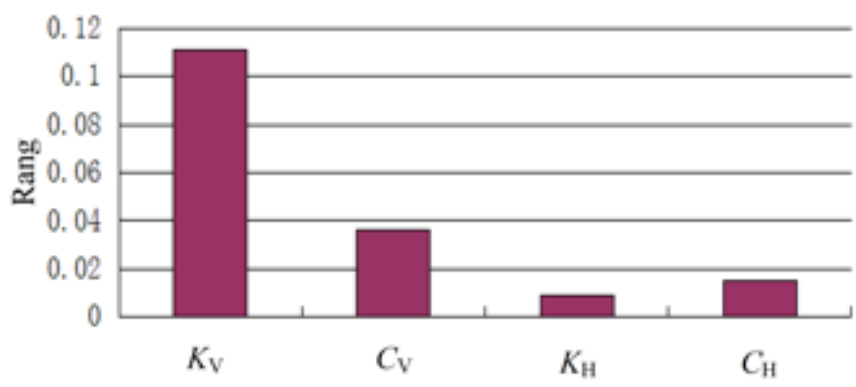

(g) Sensitivity analysis of sub-rail parameter on lateral Sperling indicator

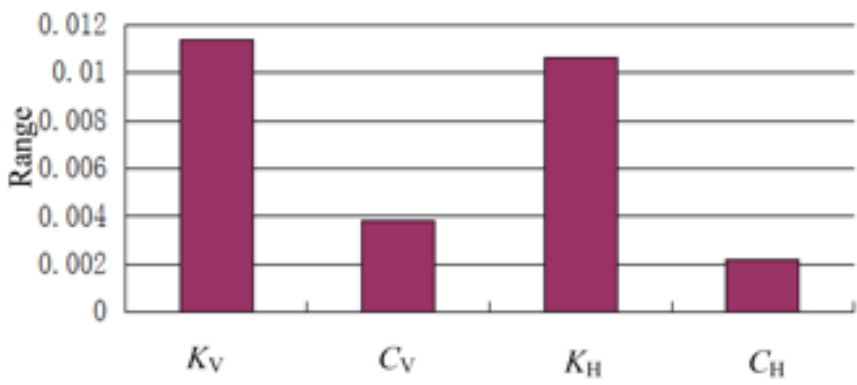

(h) Sensitivity analysis of sub-rail parameter on vertical Sperling indicator

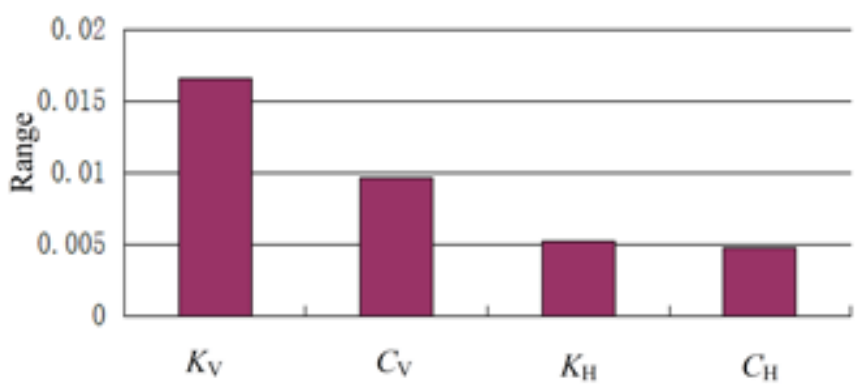

(i) Range analysis of sub-rail parameter on vehicle comfort

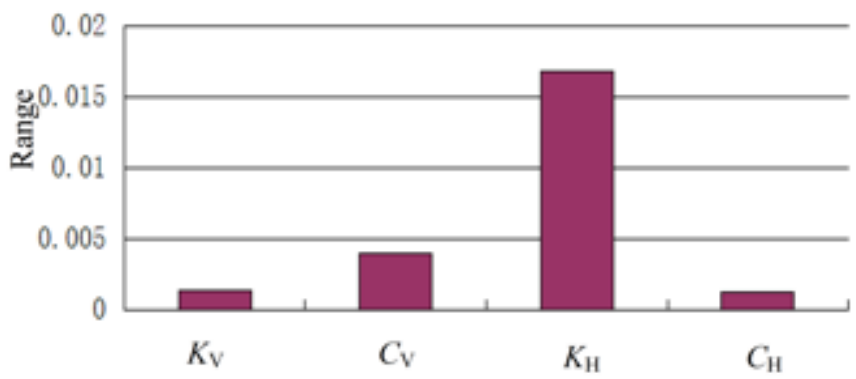

Fig. (3). Range analysis of sub-rail parameter on vehicle comfort dynamics performances.

\section{CONCLUSION}

In this paper, analysis model of the vehicle and the 60 $\mathrm{kg} / \mathrm{m}$ Rail Chinese NO.18 turnout with a movable-nose are established and an analysis has been carried out. The vehicle passes through a turnout on the divergent route with orthogonal design. The conclusion is drawn as follows: 
Table 4. Optimal parameter scheme for vehicle performance.

\begin{tabular}{|l|l|c|}
\hline \multicolumn{1}{|c|}{ Evaluation Index } & Optimum Track Mechanical Parameters & Parametric Sensitivity \\
\hline \hline Maximum Value of Lateral Wheel set Force & $\mathrm{A}_{1} \mathrm{~B}_{5} \mathrm{C}_{1} \mathrm{D}_{3}$ & $\mathrm{C} \rightarrow \mathrm{A} \rightarrow \mathrm{D} \rightarrow \mathrm{B}$ \\
\hline Maximum Value of Vertical Wheel-rail Force & $\mathrm{A}_{1} \mathrm{~B}_{5} \mathrm{C}_{3} \mathrm{D}_{1}$ & $\mathrm{~A} \rightarrow \mathrm{B} \rightarrow \mathrm{C} \rightarrow \mathrm{D}$ \\
\hline Maximum Value of Derailment Coefficient & $\mathrm{A}_{1} \mathrm{~B}_{5} \mathrm{C}_{1} \mathrm{D}_{2}$ & $\mathrm{C} \rightarrow \mathrm{A} \rightarrow \mathrm{B} \rightarrow \mathrm{D}$ \\
\hline Maximum Value of Wheel Unloading Rate & $\mathrm{A}_{1} \mathrm{~B}_{5} \mathrm{C}_{3} \mathrm{D}_{1}$ & $\mathrm{~A} \rightarrow \mathrm{B} \rightarrow \mathrm{C} \rightarrow \mathrm{D}$ \\
\hline Maximum Value of Lateral Car body Vibration Accelerations & $\mathrm{A}_{1} \mathrm{~B}_{2} \mathrm{C}_{1} \mathrm{D}_{5}$ & $\mathrm{C} \rightarrow \mathrm{A} \rightarrow \mathrm{D} \rightarrow \mathrm{B}$ \\
\hline Maximum Value of Vertical Car body Vibration Accelerations & $\mathrm{A}_{1} \mathrm{~B}_{5} \mathrm{C}_{5} \mathrm{D}_{4}$ & $\mathrm{~A} \rightarrow \mathrm{B} \rightarrow \mathrm{D} \rightarrow \mathrm{C}$ \\
\hline Maximum Value of Lateral Sperling Value & $\mathrm{A}_{5} \mathrm{~B}_{4} \mathrm{C}_{4} \mathrm{D}_{2}$ & $\mathrm{~A} \rightarrow \mathrm{C} \rightarrow \mathrm{B} \rightarrow \mathrm{D}$ \\
\hline Maximum Value of Vertical Sperling Value & $\mathrm{A}_{5} \mathrm{~B}_{5} \mathrm{C}_{5} \mathrm{D}_{5}$ & $\mathrm{~A} \rightarrow \mathrm{B} \rightarrow \mathrm{C} \rightarrow \mathrm{D}$ \\
\hline Maximum Value of Ride Comfort Index & $\mathrm{A}_{2} \mathrm{~B}_{3} \mathrm{C}_{5} \mathrm{D}_{3}$ & $\mathrm{C} \rightarrow \mathrm{B} \rightarrow \mathrm{A} \rightarrow \mathrm{D}$ \\
\hline
\end{tabular}

Table 5. Average and optimum of track mechanical parameters.

\begin{tabular}{|c|c|c|c|c|}
\hline \multirow{2}{*}{ Value } & \multicolumn{4}{|c|}{ Track Mechanical Parameters } \\
\cline { 2 - 5 } & Track Vertical Rigidity & Track Vertical Damping & Track Lateral Rigidity & Track Lateral Damping \\
\hline \hline Average & $5 \times 10^{7} \mathrm{~N} / \mathrm{m}$ & $5.5 \times 10^{4} \mathrm{Ns} / \mathrm{m}$ & $2 \times 10^{7} \mathrm{~N} / \mathrm{m}$ & $3 \times 10^{4} \mathrm{Ns} / \mathrm{m}$ \\
\hline Optimum Results & $2 \times 10^{7} \mathrm{~N} / \mathrm{m}$ & $10 \times 10^{4} \mathrm{Ns} / \mathrm{m}$ & $1 \times 10^{7} \mathrm{~N} / \mathrm{m}$ & $1 \times 10^{4} \mathrm{Ns} / \mathrm{m}$ \\
\hline
\end{tabular}

(a) Maximum Value of Lateral Wheel set Force

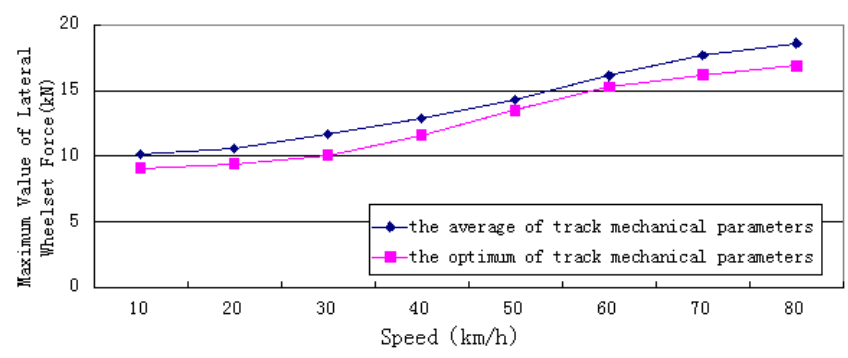

(b) Maximum Value of Vertical Wheel-rail Force

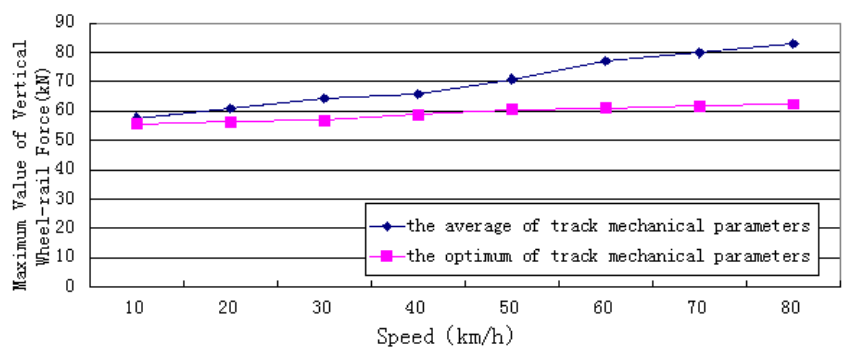

(c) Maximum Value of Derailment Coefficient

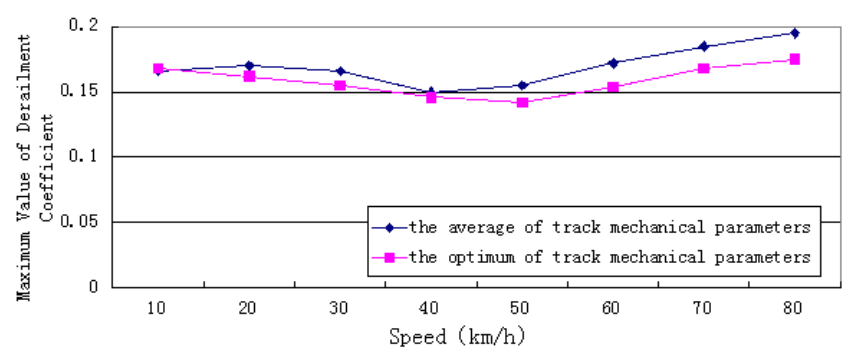

(Fig. 4) contd......

(d) Maximum Value of Wheel Unloading Rate

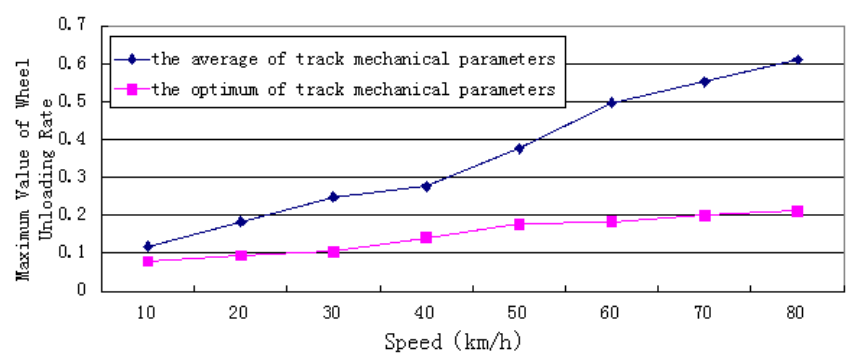

(e) Maximum Value of Lateral Car body Vibration Accelerations

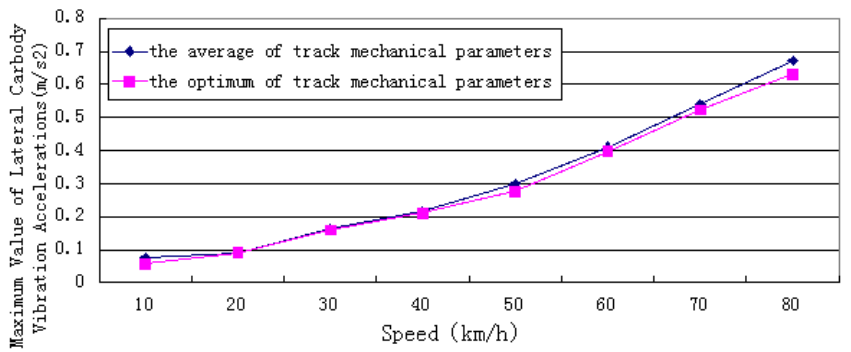

(f) Maximum Value of Vertical Car body Vibration Accelerations

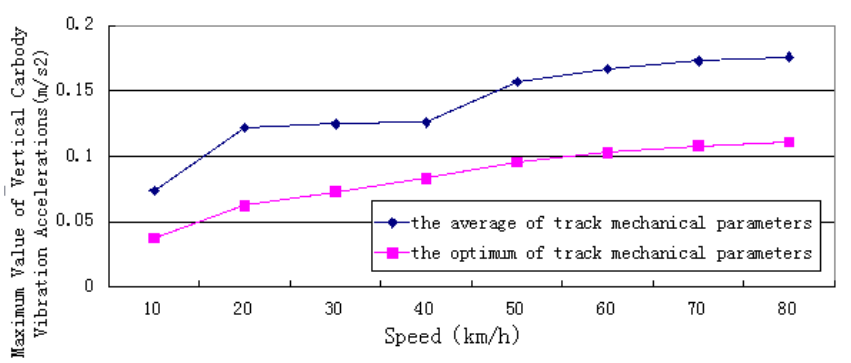


(g) Maximum Value of Ride Comfort Index

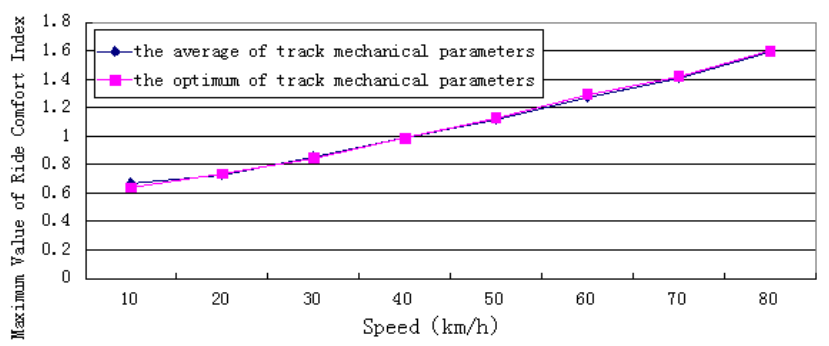

Fig. (4). Comparison results between the average and optimum of track mechanical parameters.

1. According to the orthogonal method, some simulations can be made on appropriate value of the track's mechanical and reasonable coupling parameters study.

2. From the running analysis results of vehicle on the turnout, it is revealed that the effect of track rigidity is significant, and the effect of track damping is slight. Track vertical rigidity has a significant influence on the vehicle's vertical dynamic performance and Sperling value and track lateral rigidity has an effect on vehicle's lateral dynamics performances, derailment coefficient and ride comfort index.

3. From the analysis results of orthogonal method, it is advantageous to select track vertical rigidity $2 \times 10^{7} \mathrm{~N} / \mathrm{m}$, track vertical damping $10 \times 10^{4} \mathrm{Ns} / \mathrm{m}$, track lateral rigidity $1 \times 10^{7} \mathrm{~N} / \mathrm{m}$ or $3 \times 10^{7} \mathrm{~N} / \mathrm{m}$ and track lateral damping $1 \times 10^{4} \mathrm{Ns} / \mathrm{m}, 2 \times 10^{4} \mathrm{Ns} / \mathrm{m}, 3 \times 10^{4} \mathrm{Ns} / \mathrm{m}$ or $5 \times 10^{4} \mathrm{Ns} /$. In view of economy, the optimum track lateral rigidity is $1 \times 10^{7} \mathrm{~N} / \mathrm{m}$ and track lateral damping is $1 \times 10^{4} \mathrm{Ns} / \mathrm{m}$.

\section{CONFLICT OF INTEREST}

The authors confirm that this article content has no conflict of interest.

\section{ACKNOWLEDGEMENTS}

This work was supported by the National Natural Science Foundation of China (Grant No.11102121, 51208319), Foundation of Education Bureau of Hebei Province (Grant No. Y2012013) and Natural Science Foundation of Hebei Province (Grant No. A2012210018, E2012210025).

\section{REFERENCES}

[1] Z. S. Ren, Vehicle System Dynamics, Beijing, 2007, pp. 1-62.

[2] X. C. Tan, "Study on ride comfort of the stiffness under track and dynamic characteristics of turnout", M.S. thesis, Tongji University, Shanghai, China, 2008.

[3] X. P. Chen, and P. Wang, "Distribution regularity and homogenization of track rigidity for ballastless turnout", Journal of Southwest Jiaotong University, vol. 41, pp.447-451, 2006.

[4] Y. F. Fu, and Y. Y. Luo, "Effect of stiffness under uneven turnout rail on wheel-rail dynamics system", Railway Engineering, vol 2011, pp. 139-141, 2011.

[5] K. Popp, K. Knothe, and C. Pöpper., "System dynamics and longterm behaviour of railway vehicles, track and subgrade: report on the DFG Priority Programme in Germany and subsequent research." Vehicle System Dynamics, vol. 43, no. 6-7, pp. 485$521,2005$.

[6] China Railway Baoji Bridge Group Co. Ltd., Tie Lu Dao Cha Can Shu Shou Ce, Beijing, 2009, pp.1-300.

[7] S. W. Ren, "Study on intermediate straight line and circular curve technical conditions of high-speed railway by dynamics analysis", M.S. thesis, Southwest Jiaotong University, Chengdu, China, 2012.

[8] X. C. Li, H. Z. Wang, and L. W. Wang, Experiment Design and Optimization, Beijing, 2002, pp. 105-182.

[9] R. H. Sun, X. Y. Yi and Q. S. Liu, "Mathematical Statistics", Chongqing, 2000, pp. 30-60

[10] X. P. Chen, "Study on Theory and Application of Track Stiffness in High-Speed Turnouts" Ph.D. thesis, Southwest Jiaotong University, Chengdu, China, 2008.

(C) Yongjie et al.; Licensee Bentham Open

This is an open access article licensed under the terms of the Creative Commons Attribution Non-Commercial License (http://creativecommons.org/licenses/by-nc/4.0/) which permits unrestricted, non-commercial use, distribution and reproduction in any medium, provided the work is properly cited. 